Case Report

\title{
Anaplastic ependymomas, report of 3 pediatric cases. update on molecular markers - risk groups and therapeutic options
}

\begin{abstract}
Ependymomas are neoplasms of the central nervous system (CNS) that are classified in grades I to III. They represent $10 \%$ among CNS neoplasms and increase up to $30 \%$ in the population under 3 years. The origin is postulated in the cells of the radial glia. Pathology studies, biomolecular and genetic tests have established biological differences between ependymomas according to anatomical location, risk groups have been classified that associate molecular genetic information with epidemiological variables and have facilitated a better stratification of patients. Treatment is surgery with complementary radiotherapy. The purpose of this article is to present three cases of anaplastic ependymomas (AE) that are relevant due to the low frequency of presentation among CNS tumors, to review the genetic and biomolecular markers that grouped with clinical epidemiological variables are impacting the therapeutic schemes.
\end{abstract}

Volume 8 Issue 5 - 2017

\author{
Sergio Cafiero,' Fernando González Trujillo, ${ }^{2}$ \\ Nohelia Muñoz, ${ }^{3}$ Nicolás Duran, ${ }^{4}$ Paola \\ Andrea Posada España, ${ }^{5}$ Walter Pineda \\ Perez $^{6}$ \\ 'Chief of the Radiation Oncology department, Centro integral \\ del cáncer, Clínica de Occidente, Colombia \\ ${ }^{2}$ Clinical Neurology Observer in Neuro-Oncology, Centro \\ integral del cáncer, Clínica de Occidente, Colombia \\ ${ }^{3}$ Pathologist, Centro integral del cáncer, Clínica de Occidente, \\ Colombia \\ ${ }^{4}$ Radiologist, Centro integral del cáncer, Clínica de Occidente, \\ Colombia \\ ${ }^{5}$ Physician, Fundación Universitaria San Martin, Colombia 6 \\ Technician in Radiology, Clínica de Occidente, Colombia
}

\author{
Correspondence: Sergio Cafiero, Chief of the Radiation \\ Oncology department, Centro integral del cáncer, \\ Clínica de Occidente, Colombia, \\ Email cafieroserigo@hotmail.com
}

Received: August 24, 2017 | Published: September 2I, 2017

\section{Introduction}

Ependymomas are central nervous system (CNS) neoplasms classified according to the World Health Organization (WHO) in grades I to III (benign to undifferentiated with anaplastic changes), according to statistics, they make up approximately $10 \%$ among neoplasms of the CNS and increased up to $30 \%$ in the population below 3 years, ${ }^{1}$ in childhood is the third most frequent tumor after the pilocytic astrocytoma and medulloblastoma. ${ }^{2}$ The origin is postulated in the cells of the radial glia. ${ }^{2}$

Pathology studies supplemented with biomolecular and genetic tests allowed to establish that there are biological differences between ependymomas according to anatomical location, risk groups have been classified that associate molecular genetic information with epidemiological variables that has facilitated to better stratify patients. ${ }^{1,2}$. Treatment is surgery with complementary radiotherapy; chemotherapy has not impacted the tumor control rates; although, they have been improved. Epidemiological variables in quantity are not relevant, progression-free survival for ependymoma hardly reaches $60 \%$ at 5 years. ${ }^{1}$ Genetic and biomolecular development allowed to establish markers with a prognostic impact that have allowed to classify patients in risk subgroups, that lets to define better the therapeutic interventions. ${ }^{2}$

The purpose of this article is to present three cases of anaplastic ependymomas (AE) that are relevant due to the low frequency of presentation among CNS tumors, to review the genetic and biomolecular markers that grouped with clinical epidemiological variables are impacting the therapeutic schemes. Create concern in oncologic study groups to complement the findings of pathology with genetic and biomolecular studies to better classify patients and perform more individualized therapeutic interventions.

\section{Clinical cases}

\section{Case report no. I}

A 4-year-old male patient, who consulted the emergency department for a seizure episode lasting less than 5 minutes, was treated with Diazepam and Phenytoin impregnation. Once the patient stabilized, he was alert, obeyed orders, without any motor or sensory deficit. The medical history was not relevant. Initial brain images by CT and MRI revealed a supratentorial brain tumor, adjacent to the occipital horn and to the right lateral ventricle, a hypodense zone with a discrete mass effect on the occipital horn of the adjacent lateral ventricle. The tumor was removed by surgical approach, by right occipital route. Postoperative control resonance reported the resection area in the right occipital lobe with gliosis and uptake at the edges of resection, with no nodular uptake suggesting no residual tumor, with thickening and meningeal uptake in the interhemispheric region to the right side and with a small non-compressive subdural haematoma; Spinal extension resonances did not show leptomeningeal dissemination. The pathologic diagnosis described a tumor with monomorphic cells of round and oval hyperchromatic nuclei, forming perivascular pseudorosettes, with mitosis and necrosis, compatible 
with anaplastic ependymoma (Figure 1). The radiotherapy oncology service referred the tumoral pathology as an anaplastic ependymoma with poor prognosis, not being a candidate for chemotherapy. It was programmed for cranial spinal radiation therapy, received doses of 5940 CGY (centigrays) in fractions of 180CGY. The patient's followup period up to 18 months did not show tumor recurrence, the last brain resonance reported encephalomalacia in the right occipital lobe with occipital horn enlargement by retraction, no contrast uptake suggestive of tumor recurrence; the spinal expansion resonances were normal. The patient consulted several times for tonsillitis, otitis, headache and dizziness. Always with improvement and departure.

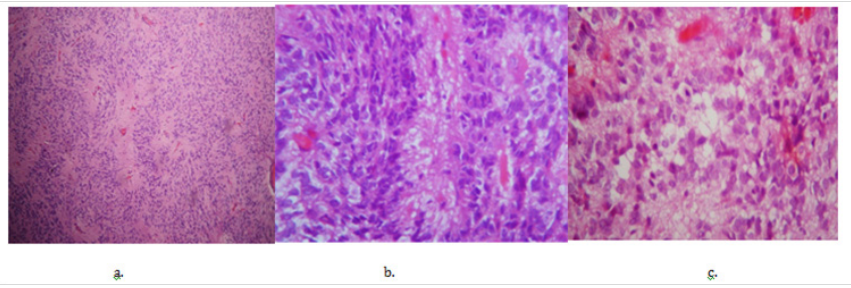

Figure I Pathology:Anaplastic Ependymomas.

a. Monomorphic cells of round and oval hyperchromatic nuclei, forming perivascular pseudorosettes, with frequent mitosis and necrosis.

b. Arrangement of pseudorosettes with perivascular palisade (Coloration H\&E I0x).

c. Frequent mitosis and necrosis (Coloration H\&E 40x).

\section{Case report no. 2}

A 15-year-old male patient who consulted for a 5-month headache that increased with valsalva maneuvers, photophobia, located in the occipital region. The physical examination revealed an ataxic syndrome. Brain tomography and MRI imaging showed an infratentorial brain tumor in the posterior fossa, compromising the IV ventricle, approximately $4.5 \times 3 \mathrm{~cm}$, with heterogeneous enhancement with Gadolinium, the lesion expands and obliterates the IV ventricle, extending through the holes towards the area of the right pontocerebellar angle; produces supratentorial obstructive hydrocephalus with transependimary exudation; no spinal spread was found (Figure 2a). The tumor was completely removed by sub-occipital craniectomy. Postoperative control resonance showed recession of the right cerebellar amygdala, post-operative changes without abnormal contrast uptake, and no residual tumor lesion was found. The pathological diagnosis was anaplastic grade III ependymoma, monomorphic cells of round and oval nuclei were observed, forming perivascular pseudorosettes, with frequent mitosis and necrosis (Figure 1). Subsequently, radiotherapy was performed with IMRT with a total dose of 5400 CGY. The patient finished the treatment without neurological deficit. The patient remains in followup with a stable condition.

\section{Case report no. 3}

A 1-year-old male patient who consulted for swallowing disorder, sobbing spasm, he fell down and later presented torticollis, was taken to the emergency department, performed brain tomography and MRI that evidenced tumoral lesion in the posterior fossa that compromises left cerebellar hemisphere, which measures $2.8 * 1.6 \mathrm{~cm}$, heterogeneous, with calcifications, enhances with contrast and compresses the brain stem deforming the IV ventricle; magnetic resonance imaging of the cervical spine did not show tumor spread (Figure 2b). Surgery was performed with incomplete resection of $70 \%$. The post-surgical neurological state was a patient with hypoactivity, involvement of
VII, IX, X, XI cranial pairs, swallowing disorder, left hemiparesis and cerebellar syndrome. The evolution was regular, the control resonance showed the surgical history and the presence of 2 residual oval lesions, the largest located near the IV ventricle, with compression of the cerebral stem and the second located in the cerebellar hemisphere. The second surgical intervention was performed, infiltrating lesions were observed in the IV ventricle and Lushka foramen, complete resection was achieved. The pathological diagnosis was recurrent ependymoma, with findings of monomorphic cells of round and oval hyperchromatic nuclei, forming perivascular pseudorosettes, frequent mitosis and necrosis compatible with anaplastic ependymoma (Figure $1)$.
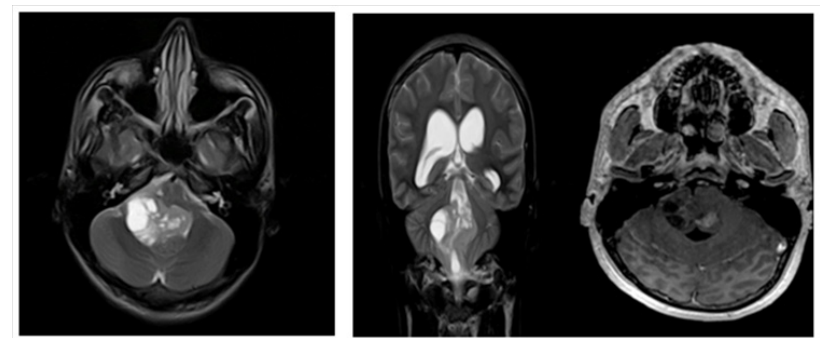

Figure 2a Radiology:Anaplastic Ependymomas.

Patient no. 2. Lesion with cystic and solid component, presenting compromise of the fourth ventricle, the uptake is heterogenous with nodular component.

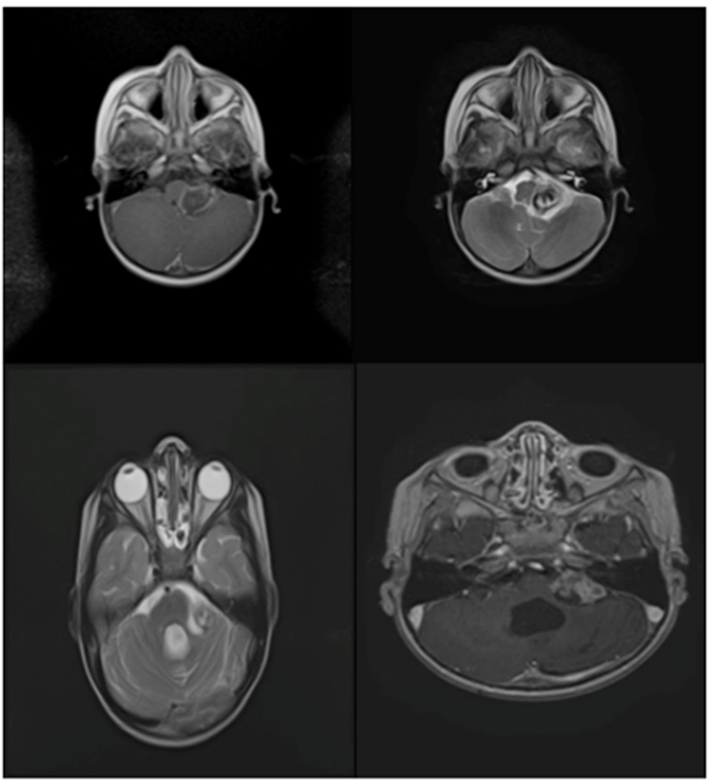

Figure 2b Radiology:Anaplastic Ependymomas.

b. Patient No.3. Pre-surgical and post-surgical axial T2 sequences, a tumor presenting calcifications, adjacent to the fourth ventricle, the uptake is heterogeneous with annular predominance. In the post-surgical control brain resonance, a residual lesion is observed, with persistent uptake of annular predominance.

Complementary treatment was done with radiotherapy and chemotherapy. Radiotherapy was performed, with doses of $54 \mathrm{GY}$ in 30 fractions of $1.8 \mathrm{GY}$, with photons of $6 \mathrm{mev}$, with IMRT technique, with multi-leaf collimator during 30 sessions. The chemotherapy was with the protocol for anaplastic ependymoma "Baby Pog III modified" with Vincristine, Etoposide, Cyclophosphamide, Cisplatin and Mesna rescue, receiving 4 courses. A control brain resonance imaging was made, showing a reduction of the initial residual lesion from $2.4 \times 1.7$ $\mathrm{cm}$ to $1.6 \times 1 \mathrm{~cm}$. 
The patient presented episodes of febrile neutropenia after chemotherapy with lung infection and required antibiotics. The patient was followed up on an outpatient basis over a period of 19 months, the last brain resonance study showed lesion progression up to $2.7 \times 2.3 \times$ $2.9 \mathrm{~cm}$ with contrast uptake, in brain perfusion sequences an increase in blood flow and volume was reported, which means a progression of the lesion. He did not receive other interventions, he continued in palliative care.

\section{Review}

The Ependymoma is a heterogeneous neoplasm with histological and genetic diversity. The WHO classification includes pathological variations of ependymoma grouped like this: grade I with Myxopapillary and subependymoma variant, the classic tumor grade II (clear cells, tanacitic and papillary variants) and grade III anaplastic type. There are reported differences between infratentorial and supratentorial ependymomas, it was observed that the pathological classification does not correlate with the anatomical location and the clinical results. ${ }^{1}$. Relevant cytogenetic abnormalities found in anaplastic ependymomas (AE) are the 1q chromosome gain, anomalies in copies of chromosomes 6q25 (LATS1), 9p21 (CDKN2A), 11q.13 (generates the fusion protein C11ORF95-RELA). A group was established that included patients with the following clinical-molecular profile: very young by age, recurrent tumor located in the posterior fossa and the $1 \mathrm{q}$ gain that is related - independently- with a worse prognosis and a strong predictor of adverse outcomes. ${ }^{1,2}$ The alteration in the chromosomes 6q25 (LATS1), 9p21 (CDKN2A) was found infrequently. They are related in several studies with variable results. Currently the alteration in chromosome 11q.13 generated interest, there is a fusion protein called C11ORF95-RELA that enters the cell nucleus activating genes related to the NF-KB pathway (nuclear factor kapa beta) generating changes in neural stem cells precursors of ependymal cells; was more reported in supratentorial ependymomas. ${ }^{3-5}$

Classical Ependymomas have histological characteristics that demonstrate a solid tumor, with non-infiltrative growth pattern, presence of perivascular pseudorosettes (processes of tumor cells that are organized radially surrounding a blood vessel); the $\mathrm{AE}$ include other findings like hypercellularity with nuclear hyperchromy and / or nuclear pleomorphism, abundant mitosis, microvascular proliferation and "pseudopalysed" necrosis. The presence of 2 or more characteristics are indicative of an $\mathrm{AE}$ and is associated with short survivals. ${ }^{5}$. The heterogeneity of ependymomas related to differences by supratentorial or infratentorial anatomical location, pathological phenotypes and distinct cytogenetic abnormalities require different therapeutic approaches. The current reference treatment is maximum surgical resection, followed by radiotherapy. ${ }^{1,6}$ For supratentorial tumors that infiltrate the adjacent brain, total or enlarged resection with the least possible tumor residue is recommended; with those of posterior fossa the complete resection is limited by the adjacent structures. ${ }^{1}$. The second surgery when there is recurrence has proven to be safe. ${ }^{6}$ Complete surgical resection allows radiotherapy to be deferred in many cases in patients younger than 3 years, where radiotherapy causes significant side effects. ${ }^{6}$

The diagnostic imaging is based mainly on magnetic resonance for its anatomical resolution, usually are hipointense in T1 sequences, high signal in $\mathrm{T} 2$ sequences and intermediate in FLAIR sequences, the presence of calcifications is determined with the echo gradient sequences, as well as the hemorrhagic content. Diffusion sequences are also non-specific and a large number of tumors may not exhibit restriction to the diffusion. ${ }^{6,7}$. Differentiation with astrocytomas and other tumors is based on their localization and morphology since the appearance in different sequences and uptake of contrast is similar in most lesions. ${ }^{6}$. Radiotherapy is applied in addition to surgery. When complete tumor resection is achieved, it is applied to the tumor bed and in the peri-lesional margin up to $2 \mathrm{~cm}$, for leptomeningeal dissemination, cranio-spinal radiotherapy is preferred. The conformational radiotherapy technique is effective for well localized posterior fossa tumors with complete resection. In patients younger than 3 years, the tendency is to omit or delay radiotherapy. The suggested doses of radiotherapy given for tumors with complete infratentorial resections are 59.4 Gy and for supratentorial 55.8 Gy. The cases with incomplete resection are applied 59.4 Gy without considering the location.?

Chemotherapy is offered as an alternative in very young children to defer radiotherapy and avoid side effects in cognitive, endocrine areas. ${ }^{1,6-8}$ The results obtained with chemotherapy are regular, agents such as cisplatin are the most used with responses referred up to $30 \%$. Combined schedules of Vincristine, cyclophosphamide. There are research studies that propose schemes of pre-irradiation chemotherapy with platinum or with alkylating agents for tumors with incomplete resections. $^{7}$

\section{Discussion}

Ependymal tumors in the general population are of low presentation, it was considered important to refer to the cases studied in the institution, more, when they are of the anaplastic type, a subtype that has low reports in the world literature. ${ }^{1,9}$ Patients belong to the pediatric age group, where it is reported that it is the third most frequent tumor on presentation, ${ }^{2}$ with preference for affecting patients younger than 4 years, 2 of the patients are in the age group: Young people tend to present the most aggressive tumors compared to other age groups. ${ }^{9}$, the third patient who is 1 year old is very representative, had a severe clinical involvement with a recurrent tumor, compared to the other cases. Survival in AE is low; referenced patients have a survival period greater than 18 months, one with evidence of tumor progression. ${ }^{1,10}$

The experience reported by working groups allowed us to establish that the anatomical location of these tumors makes a difference in clinical outcomes and, to a lesser proportion, the findings of pathology, the concept refers not only to the ease of surgical approach for those located at supratentorial level (which are feasible for complete resection) compared to infratentorial ones improving survival outcomes, but, extending to biomolecular expression, which is different by anatomical location alone, with different clinical and prognostic results.

The anatomical location in the infratentorial fossa in the third patient made difficult the extensive resection by the focal infiltrative process towards neighboring structures, compromising more the patient's functionality. The pathologic findings for AEs establish more aggressive tumor subtypes that complemented with genetic and biomolecular studies (review more relevant markers in the review section) amplify the information that allows to better define the factors of prognostic type, to individualize treatments, in the sample of patients it was not possible to typify the markers to establish their relation with clinical pathological findings. , $^{2,9}$

When surgery does not achieve complete resection of the tumor as happened in 2 of the 3 cases, it is proposed to plan a second intervention and offer complementary schemes with radiotherapy, it is the therapeutic option with evidence of better results compared to 
chemotherapy. ${ }^{7,9-13}$ Early administration of post-surgery radiotherapy versus delayed tumor progression has shown improved survival and progression-free survival. ${ }^{12,13}$ All three cases received radiotherapy at the doses indicated by the protocols, with survival already referenced. The third case received a chemotherapy scheme, according to the protocols suggested by oncology groups, based on Cisplatin, regimen that has shown the best results. ${ }^{71}$ A transitory partial response was achieved with reduction in tumor residue volume; this result supports what is referenced by the literature, regarding the little impact that chemotherapy offers in tumor control.

\section{Conclusion}

The AEs are tumors with low presentation in the population, a factor that has limited the improvement of their study. The anatomical location defines differences between the AEs with clinical results and different prognoses, which must be recognized by the treatment groups. Histological diagnosis should always be complemented by genetic and biomolecular studies to better understand the tumor, define prognostic impact factors and guide therapy strategies..$^{15}$ In the near future, it is expected that the biomolecular information will allow to work in the development of the so-called "target therapies", which are already applying cancer groups around the world, which allow the application of individualized and specific therapies in the patient, complementary to the traditional schemes of surgery, radiotherapy and chemotherapy.

\section{Conflicts of interest}

There is no conflict of interest.

\section{Acknowledgements}

None.

\section{Funding}

None.

\section{References}

1. Catherine Godfraind, Joanna M Kaczmarska, Mehmet Kocak, et al. Distinct disease-risk groups in pediatric supratentorial and posterior fossa ependymomas. Neuropathol Minutes. 2012;124(2):247-257.

2. Hendrik Witt, Andrey Korshunov, Stefan M Pfister, et al. Molecular approaches to ependymoma: the next step(s). Curr Opin Neurol. 2012;25(6):745-750.
3. Cachia D, Wani K, Penas-Prado M, et al. C11orf95-RELA fusion present in a primary supratentorial ependymoma and recurrent sarcoma. Brain Tumor Pathol. 2015;32(2):105-111.

4. Pietsch T, Wohlers I, Goschzik T, et al. Supratentorial ependymomas of childhood carry C11orf95-RELA fusions leading to pathological activation of the $\mathrm{NF}-\mathrm{\kappa B}$ signaling pathway. Acta Neuropathol. 2014;127(4):609-611.

5. Christine Fuller, Adekunle M Adesina. Ependymoma Pathology. 2014.

6. Sunanda Pejavar, Mei-Yin Polley, Sarah Rosenberg-Wohl, et al. Pediatric intracranial ependymoma: the roles of surgery, radiation and chemotherapy. J Neurooncol. 2012;106(2):367-375.

7. Tobey J MacDonald, Max J Coppes. Pediatric Ependymoma Treatment \& Management. Medscape. 2014.

8. Riccardo Soffietti, Chiara Bosa, Luca Bertero, et al. Temozolomide for recurrent intracranial ependymoma of the adult: patterns of response, survival and correlations with mgmt promoter methylation. Neurooncology. 2014;16:42-52.

9. Wright KD, Gajjar A. Current Treatment Options for pediatric and adult Patients With ependymoma. Current Treatment Options in Oncology. 2012;13:465-477.

10. Villano jl, Parker ck, Dolecek ta. Descriptive epidemiology of ependymal tumours in the United States. Br j cancer. 2013;108(11):2367-2371.

11. Cage TA, Clark AJ, Aranda D, et al. A systematic review of treatment outcomes in pediatric patients with intracranial ependymomas. $J$ Neurosurg Pediatr. 2013;11(6):673-681.

12. Liu AP, Shing MM, Yuen HL, et al. Timing of adjuvant radiotherapy and treatment outcome in childhood ependymoma. Pediatr Blood Cancer. 2014;61(4):606-611

13. Zosia Chustecka. Radiation in Children With Ependymoma Improves Outcomes. American Society for Radiation Oncology (ASTRO) 57th Annual Meeting. 2015.

14. Sung KW, Lim do H, Lee SH, et al. Tandem high-dose chemotherapy and autologous stem cell transplantation for anaplastic ependymoma in children younger than 3 years of age. J Neurooncol. 2012;107(2):335342 .

15. Esparza SD, Sakamoto KM, Mitton BA, et al. Childhood Cancer Genetics. 2016. 